Marcello lenca

\title{
3 The Neuroenhancement Continuum and the Minimal Rule
}

\subsection{Introduction}

This chapter argues that pharmacological nootropics should not be seen, from an ethical standpoint, as qualitatively different from other activities such as healthy diet, sleep, education, mental and bodily exercise, parental care, and information technology. In contrast, it suggests that nootropic-induced enhancement should be viewed in continuity with other nonpharmacological activities through which "our uniquely innovative species tries to improve itself" (Greely et al. 2008). My argument proceeds as follows: first, I provide an overview of various forms of neuroenhancement and discuss their impact on cognitive functioning; second, I argue that forms of enhancement that have similar effects on cognitive functioning should have similar levels of ethical permissibility regardless of the physical medium through which they are administered. Since nootropics and other neuroenhancers have qualitatively analogous effects on cognitive functioning, I conclude that they should be seen as part of the same moral continuum.

Of course, claiming that different classes of neuroenhancers belong to the same functional and moral category does not imply that they are equivalent in every respect. It is reasonable to object, for instance, that enhancement by nutrition and nootropics is metabolically different than, say, mental exercise, as it involves a change in what we ingest. However, such intercategorical differences are equally distributed across the whole neuroenhancement continuum and do not justify the dichotomy between nootropics vs nonnootropic enhancers. In other words, such differences do not provide sufficient ground for justifying a priori (i.e., based on theoretical deduction rather than empirical observation) contrasting moral judgments between nootropics and all other neuroenhancers.

My argument proceeds as follows. In order to justify a priori contrasting moral judgments between the class of nootropics $(\mathrm{N})$ and that of all other enhancers (OEs), there must be at least one morally relevant property $\mathrm{P}^{1}$ that is peculiar to $\mathrm{N}$, i.e., which is a property of $\mathrm{N}$ without being a property of $\mathrm{OE}$ as well. But there is no morally relevant property $\mathrm{P}^{1}$ peculiar to $\mathrm{N}$. Therefore, a contrasting moral judgment between $\mathrm{N}$ and $\mathrm{OE}$ cannot be justified $a$ priori. And consequently, $\mathrm{N}$ and $\mathrm{OE}$ are a priori morally equivalent. In addition, I propose a minimal rule for the administration of neuroenhancing drugs and test the application of this rule in relation to a number of nootropics. This rule aims at providing guidance to physicians during the prescription of nootropics, as well as to policy bodies during the regulation of neuroenhancers. 


\subsection{Nootropics and the origin of human neuroenhancement}

The history of neuroenhancement dates back to the origin of human civilization. Written evidences and archeological tests prove that, at least since the beginning of the Bronze Age, many different cultures developed strategies to augment or extend some core mental capacities. In premodern societies, enhancement was usually obtained through both exercise techniques and the consumption of natural extracts of neuroenhancing substances, such as Ginkgo biloba, ginseng, cocaine, guaranà, yerba maté, coffee, yaupon holly, and many others (Lloyd 1911). It is also known that some epoch-making advances in the biological and cultural evolution of the human species, such as the origin of verbal language and tool use, the achievement of a metabolically adequate nutritional regime, the invention of writing, and the institution of education systems for children, exerted a massive positive feedback on our mind's functioning (Barkow et al. 1995). Nevertheless, ethical concerns about neuroenhancement have arisen only in the past 2 decades after some pharmaceutic psychostimulant drugs, chiefly analeptics such as Ritalin and Modafinil, have been invented and widely marketed.

The term nootropic (from the Greek voũ “ "mind” and Trépein “to turn”) was coined in 1972 by the Romanian psychologist and chemist Corneliu E. Giurgea to refer to the whole set of pharmaceutical drugs, supplements, nutraceuticals, and functional foods that improve processes such as attention, memory, concentration, intelligence, motivation, perception, and decision-making (Giurgea 1973).

Nootropics usually work in three possible ways: (i) by altering the availability of the brain's supply of neurochemicals, such as neurotransmitters, hormones, and enzymes; (ii) by stimulating nerve growth; and (iii) by increasing the brain's oxygen supply. In conformity with this variation in the way they influence our nervous system, we can branch nootropics into three main families: (A) neurochemical suppliers, (B) nerve growth enhancers, (C) antioxidants and neuroprotectives. Standard classifications further subdivide these three groups into subcategories in conformity with either their cellular function or their chemical composition (Table 1).

As Table 1 shows, most nootropics fall under the first category. This depends on the fact that the most direct way for a given substance to influence neuronal functionality is affecting neurotransmitters or the components of the nervous system that use such neurotransmitters. Cholinergics, for example are substances that affect the neurotransmitter acetylcholine (a facilitator of memory formation) or components of the nervous system that use acetylcholine. Analogously, dopaminergics, the most popular subfamily of neuroenhancers (e.g., L-phenylalanine, amineptine, and methylphenidate), affect the neurotransmitter dopamine or the components of the nervous system that use dopamine. Other substances affect cognition ex negativo: rather than supplying a certain neurotransmitter, they decrease the inhibiting neurotransmitter's release. The H3-receptor, for instance, decreases the release of inhibiting transmitters, such as histamine, norepinephrine, and serotonin, and 
thus increases cognition and wakefulness. Nerve growth enhancers increase brain communication, making brain processing faster. Antioxidants prevent neural oxidative stress, thus inhibiting neuronal aging and death. Neuroprotectives protect neurons from apoptosis or degeneration, thus exerting a preservative effect against brain injury and neurodegenerative diseases of the central nervous system such as Parkinson's, Alzheimer's, schizophrenia, and stroke or by simply preserving cognition from mental deterioration.

Table 1. Overview of Nootropics

\begin{tabular}{|c|c|c|}
\hline Neurochemical suppliers & Nerve growth enhancers & Antioxidants \& neuroprotectives \\
\hline $\begin{array}{l}\text { - Cholinergics: Choline, OMAE, } \\
\text { Meclofenoxate, Huperzine A, } \\
\text { Oonepezil, } \\
\text { - Serotonergics: 5-HTP, Trytophan, } \\
\text { Pyiridoxal } \\
\text { - Dopaminercics: L-Phenylanine, } \\
\text { Biopterin, Amineptine, Bupropion, } \\
\text { Methylphenidate, MAO-B inhibitors, } \\
\text { Seligeline } \\
\text { - Histamine antagonists: Ciproxifan, } \\
\text { A- 349, A-821, ABT-239 } \\
\text { - Amphetamines: Adderall, } \\
\text { Oexedrine, Methamphetamine } \\
\text { - Adrenercics: Atomoxetine, } \\
\text { Roboxetine, Synephrine } \\
\text { - Xanthines: Caffeine } \\
\text { - Euceroics: Adrafinil } \\
\text { - Direct Hormones: Vasopressin } \\
\text { Orexin }\end{array}$ & $\begin{array}{l}\text { - Growth Enhancers: Lion's } \\
\text { Maine Mushroom, Inositol }\end{array}$ & $\begin{array}{l}\text { - Simple Antioxidants: } \\
\text { Idebenone, Melatonin, } \\
\text { Coenzyme Q-10 } \\
\text { - Chief Antioxidants: Glutathione } \\
\text { - Precursors to Antioxidants: } \\
\text { Acetylcysteine (L-Cystein) } \\
\text { - Neuroprotectives: } \\
\text { Acetylcarnitine, Apoaequorin }\end{array}$ \\
\hline
\end{tabular}

The most famous nootropics currently on the market are Ritalin (methylphenidate) and Adderall (amphetamine salt-based psychostimulant). These drugs are used primarily with therapeutic aims to treat people with cognitive difficulties such as Alzheimer's disease, Parkinson's disease, and attention deficit hyperactivity disorder (ADHD).

However, more widespread use is being found by some cross-sectional surveys, because many of the pharmaceuticals used to treat psychiatric and neuropathological conditions have also turned out to improve the performance of the healthy. In 2005, one famous survey estimated that almost $7 \%$ of students in US universities have used Ritalin and Adderall in this way, and that on some campuses, up to $25 \%$ of students had used them in the past year (McCabe et al. 2005). In Switzerland, a recent survey has revealed that $13.8 \%$ of Swiss students have used prescription or illicit nootropics to increase their learning and cognitive performance (Maier et al. 2013). 
Due to their effects on the catecholamine system, these classes of nootropics increase executive functions in patients and healthy normal people, improving their abilities to concentrate, manipulate information in working memory, and make it available for further information processing. Since its introduction in 2001, a newer drug, modafinil (an acetamide analeptic), has also shown enhancement potential. The drug was approved by the U.S. Food and Drug Administration (FDA) for the treatment of narcolepsy, shift work sleep disorder, and excessive daytime sleepiness associated with obstructive apnea. Modafinil has become very popular among healthy university students in Western Europe, especially in the U.K. While some students obtain the drug illicitly (diversion of prescribed medication), others use online pharmacies (Coveney 2011).

Attention, concentration, processing speed, and accuracy are not the only mental capacities that can be enhanced through nootropics. A modest degree of memory enhancement is also possible with the ADHD medications just mentioned, as well as with drugs developed for the treatment of Alzheimer's disease such as Aricept (Donepezil), which increase levels of acetylcholine in the brain. Many other compounds with different pharmacological actions are in early clinical trials, having shown positive effects on memory in healthy research subjects.

Pharmacological nootropics have raised massive ethical concerns and sparked debate on their moral and legal permissibility (Dees 2007; Racine and Forlini 2010; Meyers 2014). Despite this disproportionate ethical attention on pharmacological products, not all nootropics have been synthetized in the laboratory. Omega-3 fatty acids, for instance, which increase cognitive performances and decrease the risk of impairment of cognitive speed (Fontani et al. 2005), are fats commonly found in marine organisms and plant oils. Isoflavones, which are related to cognitive function, are produced almost exclusively by vegetables of the Fabaceae (i.e., Leguminosae, or bean) family. The same goes for vitamin compounds such as B vitamins, which influence cognitive function through an effect on levels of methylation and homocysteine, and vitamin $\mathrm{D}$, whose active form seems to be involved in brain development and adult brain function.

\subsection{The neuroenhancement continuum: nootropics and other enhancers}

Brain functioning can be improved not only by means of pharmaceuticals but also by nontherapeutic tools such as adequate exercise, nutrition, parental and medical care, and sleep. It is well known that all these kinds of activities can massively influence the functioning of our mind and that their deprivation causes permanent damage on the development of cognitive faculties. Nutrition is a paradigmatic example: evidence shows that undernutrition, especially at an early age, affects brain growth and intelligence quotient (IQ). For instance, the majority of students with 
the lowest scholastic achievement scores present suboptimal head circumference (anthropometric indicator of past nutrition and brain development) and brain size (Leiva et al. 2001). Recently described effects of dietary factors on neuronal function and synaptic plasticity have revealed some of the vital mechanisms that are responsible for the action of diet on brain health and mental function, therefore illuminating - at the molecular level - this causal relation subsisting between food and cognition. In particular, research in the nutrition sciences has provided exciting evidence for the influence of dietary factors on specific molecular systems and mechanisms that maintain mental function. For instance, a diet that is rich in omega-3 fatty acids is garnering appreciation for supporting cognitive processes in humans and upregulating genes that are important for maintaining synaptic function and plasticity in both humans and rodents (Fontani et al. 2005). On the other hand, diets that are high in saturated fats, common in junk food, are becoming notorious for reducing molecular substrates that support cognitive processing and increasing the risk of neurological dysfunction in both humans and nonhuman animals $(\mathrm{Wu}$ and Miller 2005). Similar conclusions can be drawn for sleep, which is needed to regenerate many cellular components of the body, especially the brain, so that they may continue to function optimally. After periods of extended wakefulness or reduced sleep, neurons may begin to malfunction, visibly affecting a person's behavior. In particular, certain stages of sleep are needed for the regeneration of neurons and glial cells within the cerebral cortex, while other stages of sleep seem to be used for forming new memories and generating new synaptic connections. The effects of sleep deprivation on behavior have been tested in relation to the presence of activity in different sections of the cerebral cortex. Results show that some brain areas can be heavily damaged from bad sleep habits. During verbal learning tests on subjects who are fully rested, for instance, functional magnetic resonance imaging (fMRI) scans show that the temporal lobe of the cerebral cortex - i.e., a brain area associated with the processing of language - is very active. However, in sleep-deprived subjects (daily sleeping hours $<5$ ), there is no activity within this region (Chee and Chuah 2008).

Research on brain development shows that similar positive effects on cognition can be attributed to education (Brayne et al. 2010), mental and physical exercise (Hillman et al. 2008), the use of information technologies (Bonavita et al. 2015), and parental care during infancy (Feldman et al. 2014).

\subsection{The neuroenhancement continuum II: cognition and other systems}

Immunology constantly seeks to enhance people's immune system in order to increase their protection against disease. In accomplishing this task, physicians make use of three classes of measures: preventive clinical measures (e.g., vaccines), therapies (e.g., antibiotics), and the promotion of nonclinical enhancers (lactobacilli, 
vitamin $\mathrm{C}$, and so on). All these measures involve significant alteration of the original system functions or architecture. For example, most people at birth are not immune to diseases such as hepatitis B, poliomyelitis, and rubella. However, in several countries, compulsory vaccination policies have enhanced baseline immunity and augmented individual and collective defenses against communicable disease. Antibiotics can rapidly cure many diseases that otherwise could lead to the patient's death. Nontherapeutic enhancers present in food and supplements can power the system and allow its better functioning. In this process of enhancing the immune system, physicians and public health promoters do not hold the assumption of a presumed default mode of the system. They rather aim at system optimization, namely, the state of the immune system in which it is most capable to protect an organism against disease. In other words, they try to indefinitely maximize the functioning of that system. The only clause that is usually put to this indefinite enhancement of the immune system is that the system augmentation should not thereby cause negative effects of comparable relevance on other related systems. The same thing can be said of the enhancement of the cardiovascular (indefinite optimization through preventive nutrients, pharmaceuticals, exercise, and implants) and the locomotion system (indefinite optimization through preventive nutrients, drugs, exercise, prosthetic limbs, and means of transport).

This paper argues that this same implicit decision-making strategy adopted by immunologists, cardiologists, and locomotion researchers should be applicable to neuroenhancement. In contrast to dichotomist classifications that attribute qualitatively different moral statuses to nootropics and nonpharmacological enhancers, this article proposes to conceptualize the various forms of neuroenhancement as a continuum.

Objections against the neuroenhancement continuum often rely on an alleged moral asymmetry between two loosely defined qualities: the artificial and the natural. Traditional cognition enhancers are seen as natural, whereas nootropics are often perceived as artificial products of laboratory research. However, the appeal to the natural as a morally relevant category is vague. As for an appeal to the artificial, the lives of almost all living humans of our time are profoundly unnatural. We live in human-built homes, wear manufactured clothes, manipulate cultivations and farming, and mediate most of our activities via technology. Furthermore, we enjoy medical care, whose invention and development have helped our species in solving many of its adaptive problems. All these aspects of our life bear very little relation to our species's natural state, however defined. To this extent, all cognition-enhancing tools with widespread moral acceptance (education, the Internet, writing, portable computers, healthy diet, mental training, and so on) are, broadly speaking, artificial. Even in the case of nutrition, which is intuitively the best candidate for naturality, most cognition-enhancing nutrients are not directly consumed in their natural form. Vitamins, for instance, are frequently consumed as supplements or artificially added to drinks and food. Similarly, iodine, which is the most important deterrent of mental 
retardation, is mostly available as an additive in cooking salt. Therefore, artificiality is not peculiar to nootropics. Saying nootropics should not be morally permissible because they are not natural would thus imply that several socially accepted substances and activities should be considered impermissible, ceteris paribus.

In the absence of solid a priori justification, neuroenhancers should be assessed through an evidence-based approach. This approach is discussed in the next section.

\subsection{A minimal rule for the administration of neuroenhancers: MiRNA}

Considering the moral equivalence between nootropics and traditional enhancers, as well as the parity principle between neuroenhancement and the enhancement of other systems of the human body, it seems unjustified to qualitatively differentiate a priori between the moral status of nootropics and that of other neuroenhancers. In contrast, I call for an evidence-based approach to the risk-benefit ratio of neuroenhancers. This ratio can be expressed in the form of a simple heuristic for supporting moral decisionmaking, which I call the Minimal Rule for Neuroenhancement Administration (MiRNA):

If something can improve one or many mental abilities, without thereby causing side effects of comparable relevance, then it is morally permissible to promote its application and diffusion.

MiRNA has three advantages. First, it allows a shift from a priori justification to evidence-based evaluation. Second, MiRNA offers a simple operational model for moral judgment concerning neuroenhancement. The rule can be expressed as follows:

$$
\mathrm{n} \in\{\mathrm{mpN} \text { iff } \mathrm{n}=b / c>1\}
$$

where $n$ is every given nootropic about which we want to make a moral judgment, $b$ is the variable computing the potential benefits of this nootropic, $c$ is the variable computing its negative side effects (costs), and $m p N$ is the set of the morally permissible nootropics.

This set can be consequently described as follows:

$$
\operatorname{mpN}=\{\forall \mathrm{n}(b / c>1)\}
$$

Third, as one can infer from its structural generality, MiRNA has the advantage of not being restricted to the sole nootropics domain, but it is rather applicable to all neuroenhancers. In this case, $n$ should be replaced with $e$ (enhancer), and the set $m p N$ with the set $m p E$, whose description is $\mathrm{mpE}=\{\forall \mathrm{e}(b / c>1)\}$. MiRNA might provide a useful framework to guide ethical decision-making in the context of an increasing variety of neuroenhancing solutions and techniques. 


\subsection{Application of the MiRNA}

MiRNA permits the indexing of nootropics and other enhancers under different categories in conformity with their degree of moral permissibility. For the general aim of this paper, three moral categories of nootropics can be distinguished: (A) permissible without restrictions, (B) permissible with restrictions, and (C) impermissible unless exceptions apply. However, it is worth remembering that the results of the application of MiRNA have a degree of variability dependent on the nootropic consumer. Therefore, it may happen that the same drug is permissible for a certain class of consumers under certain conditions and not permissible for a different one under different conditions. Note that such a degree of variability does not make MiRNA vacuous. Rather, it is a characteristic of substance administration policies. Take for instance alcohol. In most legislation systems, alcohol consumption is regulated by consumer-dependent and context-dependent variables: in the US, for instance, alcohol consumption is permissible only for subjects above the age of 21. Other variables concern the place of consumption (permissible at home or in the bar, not permissible in the workplace or in the street) and the activity during which the substance is consumed (permissible when related risks are minimized, not permissible when related risks are high such as say while driving a car).

Considering this degree of consumer- and context-dependent variability, it seems highly reasonable to index in Category A all those nootropics whose use and administration have been scientifically proven to result in potential benefits and/or cause negligible adverse effects. Substances that can be indexed under this category are iodine, many natural adaptogenics and stimulants (caffeine, beta-blockers, Bacopa monnieri), omega-3 fatty acids, isoflavones (daidzein, genistein, and glycitein), cholinergics (arecoline), some acetylcholinesterase inhibitors (sage, rosemary), some vasodilators (Ginkgo biloba), some reuptake inhibitors (coluracetam, ginsenoside sources), some nerve growth stimulators (melatonin, glutathione), and all vitamins. Nootropics indexed in this category typically display demonstrated potential benefits and have been scientifically proven by laboratorial and epidemiological studies to effectively enhance cognition. Benefits range over a wide variety of enhancements, such as neuronal antioxidation (glutathione, melatonin, rosemary), improvement in shortterm memory performance (Ginkgo biloba, caffeine, melatonin, Bacopa monnieri), long-term memory potentiation (melatonin, vitamin D, caffeine, pramiracetam), IQ improvement (omega-3, iodine), slowed rate of brain atrophy (B vitamins), cognitive speed (omega-3, caffeine), improvement in spatial working memory (isoflavones, vitamin D), improvement in visual-spatial memory and construction (isoflavones), learning (arecoline, omega-3, vitamins B and D), selective attention (Ginkgo biloba), and verbal fluency (isoflavones). In addition, these nootropics are typically characterized by having very low side effects, whose potential harm levels go from the relatively harmless (omega-3, Ginkgo biloba, sage, vitamins) to the moderately 
harmful adverse effects of little clinical importance. Reported side effects of Category A nootropics include somnolence (melatonin), insomnia (ginseng, caffeine), allergic reactions (rosemary), and hyperthyroidism (iodine). The characteristic of Category A nootropics is that these side effects are epidemiologically infrequent (affect not more than $2 \%-3 \%$ of patients) and tendentially related to substance abuse (not observed in consumption of small doses).

Under Category B are indexed all those nootropics whose administration has been scientifically proven to provide high potential benefits with an appreciable degree of unintended negative side effects. Substances that can be indexed under this category are eugeroics (modafinil, adrafinil), adrenergics (atomoxetine, reboxetine, synephrine), some dopaminergics (methylphenidate), some a2A receptor agonists (guanfacine), direct hormones (vasopressin, pregnenolone, orexin), fipexide, some racetams (oxiracetam and aniracetam), and the acetylcholinesterase inhibitor huperzine. These nootropics typically display high potential benefits and have been scientifically proven by many laboratorial and epidemiological studies to effectively enhance cognition (Turner et al. 2004). Benefits range over a wide variety of enhancements, such as enhancements of attention (oxiracetam, methylphenidate), general cognitive function (huperzine), concentration (modafinil, neuroleptics, methylphenidate), alertness (modafinil, methylphenidate), memory (oxiracetam, donepezil, pregnenolone), and scores in tests for logical performance (oxiracetam). Category B nootropics are typically characterized by having appreciable adverse effects, whose potential harm levels range between the moderate and the considerable, but still widely below the benefit levels. Reported side effects of Category B nootropics include nausea and vomiting symptoms (donepezil, nicergoline), dizziness and drowsiness (guanfacine, nicergoline, modafinil), headache (guanfacine), nervousness (methylphenidate, modafinil), and anxiety (modafinil). These side effects usually have an epidemiological rate between $2 \%$ and $13 \%$ of cases and can appear even at normal dosage.

Under Category C are indexed all those nootropics that are not scientifically proven to confer significant benefits, and/or cause high and/or disproportionate adverse effects. Nootropics indexed in this category typically do not fulfill the condition $b / c>1$. Common Category $\mathrm{C}$ nootropics are stimulant alkaloids (cocaine), nicotine, fipexide, empathogen-enactogen drugs, benzodiapezines (Restoril; Normison; Euhypnos), dissociatives (phencyclidine [PCP]), and barbiturates (Nembutal, Seconal, Amytal). These agents typically either have no experimentally significant positive effects or do have little positive effects disproportionate to the adverse effects. Reported side effects of Category $\mathrm{C}$ nootropics include fever (fipexide), hepatitis (fipexide), cardiovascular disease (heroin, dehydroepiandrosterone [DHEA]), Olney's brain lesion (PCP), coma (Nembutal, Euhypnos), and respiratory depression (Euhypnos, Normison). All these adverse effects could lead to death. In conformity with MiRNA, Category C nootropics, as they fail to fulfill the condition $b / c>1$, should not be considered morally permissible for unrestricted administration among healthy subjects. 


\subsection{Safety, self-determination, equality, and information}

Approaching the problem of cognitive enhancement from the perspective of riskbenefit ratio does not imply giving up substantive ethical concerns. On the contrary, it involves at least three substantive ethical concerns. The first concern is safety. Nootropics must not only be effective but also safe for consumers. This concern is synthetically expressed by the principle that the maximization of potential benefits must be balanced against the minimization of adverse side effects. For newly developed nootropics, two conditions must be fulfilled to ensure safety and thus minimize side effects: (i) all phases of experimental trials must be conducted wherever possible during clinical testing; and (ii) unregulated off-label administration and marketing must be prevented. Clinical trials involving new drugs are commonly classified into four phases:

1. Screening for safety, i.e., the phase in which researchers test an experimental drug or treatment on a small group of subjects to evaluate its safety, determine a safe dosage range, and identify negative side effects.

2. Establishment of the testing protocol, i.e., the phase in which the experimental study drug or treatment is extended to a larger group of subjects to further evaluate its safety and to test its effectiveness on a large scale.

3. Final testing, i.e., the phase in which the experimental study drug or treatment is given to large groups of subjects to definitively confirm its effectiveness, monitor the identified side effects, compare it to treatments already in use, and collect information that will allow safe use of the drug (or treatment).

4. Postapproval studies, i.e., the postapproval and postmarketing phase in which the risks and benefits are further monitored and the drug's optimal use is finally delineated.

For most nootropics on the market today, this fourth phase has not been fully completed. Therefore, further longitudinal studies focusing on the long-term side effects are still required. Until no conclusive results concerning long-term experimental trials are available, no precise quantification of the drug's potential side effects is possible. And, consequently, no clear benefits-costs ratio can be computed. In the absence of such evidence, the applicability of MiRNA remains limited. In fact, as long as the long-term side effects of a given nootropic $n$ are partly unknown, there is no certainty that the upfront benefits override the long-term costs.

It is worth pointing out, however, that a drug that causes serious adverse medical consequences but restores good cognitive functioning in severely ill individuals (i.e., people with advanced dementia) might be deemed safe enough to prescribe. Nonetheless, these risks would be unacceptable for healthy individuals seeking enhancement, in particular, for children. In these cases, adjustments to the parameters might be required. For example, for all cases where the threshold of risk toleration must be low or very low (as in the case of healthy children), we need to (i) 
set the value of $b / c$ as much greater than 1; and (ii) specify the value of $c$ as close to zero. The resulting rule would then be as follows:

$$
n \in\{m p M\} \text { iff } n=\frac{b}{c} \gg 1 \text {, with } c \cong 0 \text {. }
$$

The second concern is self-determination. Appropriate ethical guidelines should safeguard the rights to self-determination, mental integrity, and cognitive liberty of individuals (Ienca and Andorno 2017). With the possible exception of specific occupational figures (e.g., surgeons and airplane pilots), people must reserve the right to refute pharmaceutical enhancement. In a specular way, people willing to profit from cognitive enhancement must have the right to do it, provided (i) the nootropic in question meets all safety requirements, (ii) the subject in question is psychologically and legally responsible for his or her own decisions, hence entitled to exercise his or her right to cognitive liberty. To this extent, a special case is represented by children, dementia patients, and in general individuals who cannot be considered mentally competent. In such cases, the subject's deliberation must be balanced by a careful assessment of the medical authority, advanced directives, and legal proxies.

The third concern is equality. Considering that employers, schools, or governments should not generally require the use of cognitive enhancements in order to safeguard the right to self-determination and prevent indirect coercion, they must nevertheless guarantee that neuroenhancers are fairly distributed. Appropriate policy governing the use of cognitive enhancement should therefore avoid exacerbating socioeconomic inequalities, in addition to making nootropics as cheap and capillary distributed as possible.

The fourth concern is information. Appropriate policy should seek to increase public understanding of neuroenhancement and broadly disseminate information concerning the risks, benefits, and eventual alternatives to pharmaceutical neuroenhancement. Alternatives might include healthy nutrition, regular exercise, optimization of sleep patterns through self-monitoring, and other options in the neuroenhancement continuum. In addition, an examination of the social values and pressures (in particular, at school, in academia, and in the business and finance world) that make cognitive enhancement so attractive is highly required. This information would be provided by physicians, psychotherapists, teachers, and college health centers, similar to the way that information about healthy diet, recreational drugs, and sexually transmitted diseases is now disseminated.

\subsection{Conclusion}

This paper has argued that nootropics present no morally relevant intrinsic properties that differentiate them from other forms of enhancement, in the force of which one can (a) split the whole spectrum of neuroenhancement between nootropics vs 
other enhancers, and (b) justify a priori differential moral judgments. This drive for a priori justifications of the moral impermissibility of nootropics might not rest on epistemological but rather on psychological grounds. Indeed, the a priori refusal of nootropics might be similar to the general opposition to new techniques and technologies, a phenomenon typically prevalent during the first phases of development of such technologies. Historically observed, several techniques or technologies considered well established currently, such as vaccination, organ transplant, blood transfusion, and stem cell research, went through a similar initial opposition (Juma 2016).

In contrast to dichotomist categorizations, I have suggested that nootropics and other pharmacological neuroenhancers should be seen as part of a neuroenhancement continuum, together with several other cognition-enhancing substances and activities that include healthy nutrition, adequate sleep, education, information and computer technology, parental care, exercise, and many others.

After arguing that nootropics do not satisfy the conditions for a priori differential moral judgments compared to other enhancers, I have explored the epistemological grounds for guiding ethical decision-making with reference to nootropics and enhancers in general. In this context, I have called for an evidence-based approach to the evaluation of the risks and benefits of neuroenhancement. I represented this approach in the form of a simple decision-making heuristic, named MiRNA, in order to provide a simple and general tool for clinicians and other professionals to quickly assess the level of moral permissibility of any given enhancer. This heuristic could be advantageous for four main reasons: (a) it is applicable to all forms of enhancement, (b) it is quantitatively measurable and possibly modelizable, (c) it is computed from empirically testable variables, and (d) it might allow flexible, adaptive, and prompt decision-making.

Lastly, I applied MiRNA to a relatively wide range of nootropics currently in circulation to provide a rough index of current nootropics according to their degree of moral permissibility. This index consisted of three macro categories: (A) nootropics that are morally permissible without restrictions, (B) nootropics that are morally permissible with restrictions, and (C) nootropics that are impermissible (unless rare exceptions).

It is predictable that in the coming 20 years, many nootropics will be transferred from Category B to Category A, as advancements in neuropharmacology are gradually improving nootropics' safety and effectiveness. In managing this transfer, special attention should be reserved for the long-term effects on brain development, as there is no sufficient longitudinal evidence at the moment. In a world in which human life spans, work spans, work performances, and adaptive challenges are gradually increasing, nootropics and other neuroenhancers might become increasingly helpful for improving the quality of life, extending work productivity, as well as for mitigating normal and pathological forms of age-related cognitive decline. Safe and effective neuroenhancers will benefit not only the individual but society too. However, these benefits should be balanced with the associated risks. 


\section{References}

Barkow, Jerome H., Leda Cosmides, and John Tooby. 1995. The adapted mind: Evolutionary psychology and the generation of culture. Oxford University Press.

Bonavita, Simona, R. Sacco, M. Della Corte, S. Esposito, M. Sparaco, A. d’Ambrosio, R. Docimo, A. Bisecco, L. Lavorgna, and D. Corbo. 2015. “Computer-aided cognitive rehabilitation improves cognitive performances and induces brain functional connectivity changes in relapsing remitting multiple sclerosis patients: an exploratory study." Journal of neurology 262(1):91-100.

Brayne, Carol, Paul G. Ince, Hannah A.D. Keage, Ian G. McKeith, Fiona E. Matthews, TuomoPolvikoski, and RaimoSulkava. 2010. "Education, the brain and dementia: neuroprotection or compensation?” Brain 133 (8):2210-2216.

Chee, Michael W.L., and Lisa Y.M. Chuah. 2008. "Functional neuroimaging insights into how sleep and sleep deprivation affect memory and cognition." Current opinion in neurology 21 (4):417-423.

Coveney, Catherine. 2011. "Cognitive enhancement? Exploring modafinil use in social context." In Sociological reflections on the neurosciences. Bingley: Emerald, 203-228.

Dees, Richard H. 2007. "Better brains, better selves? The ethics of neuroenhancements." Kennedy Institute of Ethics Journal 17 (4):371-395.

Feldman, Ruth, Zehava Rosenthal, and Arthur I Eidelman. 2014. “Maternal-preterm skin-to-skin contact enhances child physiologic organization and cognitive control across the first 10 years of life." Biological Psychiatry 75 (1):56-64.

Fontani, Giuliano, F. Corradeschi, A. Felici, F. Alfatti, S. Migliorini, and L. Lodi. 2005. “Cognitive and physiological effects of Omega-3 polyunsaturated fatty acid supplementation in healthy subjects." European journal of clinical investigation 35 (11):691-699.

Giurgea, Corneliu. 1973. "The 'nootropic' approach to the pharmacology of the integrative activity of the brain 1, 2." Conditional reflex: a Pavlovian journal of research \& therapy 8 (2):108-115.

Greely, Henry, Barbara Sahakian, John Harris, Ronald C Kessler, Michael Gazzaniga, Philip Campbell, and Martha J Farah. 2008. "Towards responsible use of cognitive-enhancing drugs by the healthy.” Nature 456 (7223):702-705.

Hillman, Charles H, Kirk I Erickson, and Arthur F Kramer. 2008. "Be smart, exercise your heart: exercise effects on brain and cognition." Nature reviews neuroscience 9 (1):58-65.

Juma, Calestous. 2016. Innovation and Its Enemies: Why People Resist New Technologies. Oxford University Press.

Leiva, Plaza B., Brito N. Inzunza, Torrejón H. Pérez, Gloor V. Castro, Medina J.M. Jansana, Díaz T. Toro, Flores A. Almagiá, Díaz A. Navarro, Cáceres M.S. Urrutia, and Oltremari J. Cervilla. 2001. “[The impact of malnutrition on brain development, intelligence and school work performance].” Archivoslatinoamericanos de nutricion 51 (1):64-71.

Lloyd, John Uri. 1911. History of the Vegetable Drugs of the Pharmacopeia of the United States. JU \& CG Lloyd.

Maier, Larissa J., Matthias E. Liechti, Fiona Herzig, and Michael P. Schaub. 2013. “To Dope or Not to Dope: Neuroenhancement with Prescription Drugs and Drugs of Abuse among Swiss University Students." PLOS ONE 8 (11): e77967.

McCabe, Sean Esteban, John R. Knight, Christian J. Teter, and Henry Wechsler. 2005. "Non-medical use of prescription stimulants among US college students: Prevalence and correlates from a national survey." Addiction 100 (1):96-106.

Meyers, Chris D. 2014. "Neuroenhancement in Reflective Equilibrium: A Qualified Kantian Defence of Enhancing in Scholarship and Science.” Neuroethics 7 (3):287-298.

Moreno, Jonathan D. 2012. Mind wars: Brain science and the military in the twenty-first century. Bellevue Literary Press. 
Racine, Eric, and Cynthia Forlini. 2010. "Responding to requests from adult patients for neuroenhancements: guidance of the ethics, law and humanities committee.” Neurology 74 (19):1555-1556.

Wu, Peggy, and C. Miller. 2005. "Results from a field study: The need for an emotional relationship between the elderly and their assistive technologies". In Edited by D. D. Schmorrow, Foundations of Augmented Cognition, Vol 11. 